\title{
FRECUENCIA DE Sarcocystis sp. EN PERROS PASTORES DE ASOCIACIONES ALPAQUERAS DE MARANGANÍ, CUSCO
}

\author{
FREQUENCY OF SARCOCYSTIS SP. IN SHEEPDOGS FROM ALPACA ASSOCIATION BREEDERS, \\ Maranganí, Cusco \\ José Choque M. ${ }^{1}$, Amanda Chávez V. ${ }^{2}$, Atilio Pacheco P. ${ }^{3}$, Víctor Leyva V. ${ }^{4}$, \\ Susan Panez L. ${ }^{5}$ y Daniel Ticona S. ${ }^{5}$
}

\section{RESUMEN}

El objetivo del estudio fue determinar la frecuencia de Sarcocystis sp. en perros pastores de asociaciones alpaqueras del distrito de Maranganí, Cusco, durante las dos épocas del año (seca y lluviosa). El trabajo se realizó entre abril de 2005 y febrero de 2006. Se colectaron 211 muestras fecales de perros pastores de los productores alpaqueros de la zona. Las heces fueron analizadas mediante el método de flotación con solución de Sheather para determinar la presencia de ooquistes o esporoquistes de Sarcocystis sp. Se encontró una frecuencia de $42.3 \pm 9.2 \%$ (47/111) y $72.0 \pm 8.8 \%(72 / 100)$ en la época seca y lluviosa, respectivamente. Los resultados muestran una asociación significativa entre la tasa de infección y la época del año $(\mathrm{p}<0.05)$. El mes de noviembre presentó la mayor tasa $(77.8 \pm 12.1 \%, 35 / 49)$ y en mayo se obtuvo la menor tasa $(17.6 \pm 12.8 \%, 6 / 34)$. Además, se encontró diferencias significativas entre grupos etáreos $(\mathrm{p}<0.05)$.

Palabras clave: sarcocistiosis, alpaca, llama, canes, sexo, edad, coccidia

\section{Abstract}

The aim of the study was to determine the frequency of Sarcocystis spp. in sheepdogs of alpaca farm associations at Maranganí, Cusco, during the dry and rainy season (April 2005 until February 2006). Fecal samples $(n=211)$ were collected and evaluated using the method of flotation with Sheather solution to observe sporocysts of Sarcocystis spp. The frequency during the dry and rainy season was $42.3 \pm 9.2 \%(47 / 111)$ and $72.0 \pm 8.8 \%$ (72/100) respectively. The results showed an association between the rate of infection and the time of the year $(\mathrm{p}<0.05)$, where November had the highest rate $(77.8 \pm 12.1 \%, 35$ / $45)$ and March had the lowest $(17.6 \pm 12.8 \%, 6 / 34)$. In addition, significant difference was found due to age of the $\operatorname{dog}(\mathrm{p}<0.05)$.

Key words : Sarcocystosis, alpaca, lama, dog, sex, age, coccidia

\footnotetext{
${ }^{1}$ Práctica privada

${ }^{2}$ Laboratorio de Microbiología y Parasitología Veterinaria, FMV-UNMSM

E-mail: a_chavez_g@hotmail.com

${ }^{3}$ Laboratorio de Sanidad Animal, FAZ-UNSAAC

${ }^{4}$ Laboratorio de Reproducción Animal, FMV-UNMSM

${ }^{5}$ Estación Experimental del Centro de Investigaciones IVITA-Maranganí, FMV-UNMSM
} 


\section{INTRODUCCIÓN}

La crianza de camélidos sudamericanos (CSA) constituye una actividad pecuaria representativa del Perú, habiendo alrededor de 3’156,101 alpacas y 1'189,650 llamas (INIA, 2007). Esta actividad se desenvuelve de manera armónica en las zonas altoandinas, donde se aprovecha los pastizales naturales para la crianza y la producción de fibra y carne de CSA. No obstante, estas ventajas se ven limitadas por diversos factores que inciden negativamente en la producción, donde las enfermedades parasitarias tienen un rol muy importante $y$, dentro de ellas, la sarcocistiosis, cuyos agentes principales son Sarcocystis lamacanis y S. aucheniae (Leguía y Casas, 1999).

El Sarcocystis sp. es un protozoo intestinal, cuyo ciclo biológico es de tipo heteroxeno que requiere de la presencia de un hospedero definitivo (carnívoro) y un intermediario (herbívoro). El carnívoro, luego de consumir carne infectada desarrolla en el intestino delgado la fase sexual del parásito, liberando los ooquistes infectivos al medio ambiente. Estos son consumidos por el hospedero intermediario (ovino, bovino, equino, CSA, entre otros) desarrollándose la fase asexual del parásito en diferentes tejidos, que concluye con la formación de quistes, mayormente en los músculos, aunque a veces se hallan en el cerebro. El tamaño de los quistes varía según la especie, desde unos cuantos milímetros hasta un centímetro de longitud (Soulsby, 1988).

E 1 perro (Canis familiaris) es el hospedero definitivo de $S$. aucheniae y $S$. lamacanis y, por tanto, constituye la fuente contaminante de pastos al eliminar ooquistes $\mathrm{u}$ esporoquistes con sus heces. Estudios recientes demuestran que perros infectados experimentalmente con macroquistes de $S$. aucheniae pueden llegar a eliminar más de 37 mil esporoquistes por gramo de heces por animal en los 29 días post-infección (Cornejo et al., 2007). Además, estas habilidades biológicas sumadas al precario manejo sanitario de los productores y el papel fundamental de los perros pastores en las faenas laborales en campo, permite que se favorezca la diseminación de la sarcocistiosis. Se tienen prevalencias altas, que en muchos casos alcanza el $100 \%$ en animales mayores de 2 años de edad (Mostajo, 1983); sin embargo, se desconoce la situación de la sarcocistiosis canina en campo. Por tanto, el objetivo de este estudio fue determinar la frecuencia de la sarcocistiosis canina en asociaciones alpaqueras del distrito de Maranganí, Cusco, así como el efecto de las variables época, sexo y edad en su presentación.

\section{Materiales y Métodos}

El trabajo de investigación se desarrolló en las asociaciones alpaqueras de Chillihua, Quenamari y Tañihua del distrito de Maranganí, provincia de Canchis, región Cusco, ubicadas entre los 4,200 y 4,900 msnm. Los análisis fueron realizados en la Estación Experimental del Centro de Investigaciones IVITA-Maranganí, perteneciente a la Universidad Nacional Mayor de San Marcos y ubicada a 3,813 $\mathrm{msnm}$. La temperatura máxima varió entre 13 y $14^{\circ} \mathrm{C}$ y la mínima entre 5 y $2{ }^{\circ} \mathrm{C}$, y el promedio de precipitación anual fue de 965 mm (SENAMHI, 2005). Se considera como época lluviosa los meses de octubre a marzo y como época seca de abril a setiembre. El estudio se realizó durante los meses de abril de 2005 a febrero de 2006.

Se colectó muestras fecales de perros (Canis familiaris) de diferentes edades y sexo, que eran usados en las faenas de pastoreo. Las heces se depositaron en bolsas plásticas debidamente rotuladas y almacenadas en cajas térmicas refrigeradas para su transporte hasta el Laboratorio de Parasitología de la Estación Experimental del Centro de Investigaciones IVITA-Maranganí, para su respectivo análisis. Se realizaron 7 muestreos a los largo de los 11 meses de estudio: cuatro entre los meses de seca (mayo 
Cuadro 1. Frecuencia de Sarcocystis sp. en perros pastores de asociaciones alpaqueras del distrito de Maranganí, Cusco (2005-2006)

\begin{tabular}{llccc}
\hline & \multirow{2}{*}{ Variables } & Animales & \multicolumn{2}{c}{ Animales positivos } \\
\cline { 3 - 5 } Sexo & & & $\mathrm{n}$ & $\% \pm \mathrm{IC}^{1}$ \\
\hline \multirow{3}{*}{ Grupo etáreo (años) } & Hembra & 170 & 94 & $55.3 \pm 7.5$ \\
& Macho & 41 & 25 & $60.9 \pm 14.9$ \\
& $<1$ & 69 & 31 & $44.9 \pm 11.7$ \\
Época & $>3$ & 67 & 37 & $55.2 \pm 11.9$ \\
& Seca & 75 & 51 & $68.0 \pm 10.6$ \\
& Lluviosa & 111 & 47 & $42.3 \pm 9.2$ \\
& Abril & 100 & 72 & $72.0 \pm 8.8$ \\
Mes del año & Mayo & 34 & 6 & $46.1 \pm 27.1$ \\
& Julio & 41 & 6 & $17.6 \pm 12.8$ \\
& Setiembre & 23 & 19 & $46.3 \pm 15.2$ \\
& Noviembre & 45 & 35 & $69.6 \pm 18.8$ \\
& Enero & 40 & 26 & $67.8 \pm 12.1$ \\
\hline Total & Febrero & 15 & 11 & $73.3 \pm 22.4$ \\
\hline
\end{tabular}

${ }^{1}$ Intervalo de confianza del $95 \%$

a setiembre) y tres entre los meses lluviosos (noviembre a febrero).

La presencia de ooquistes y esporoquistes en las heces se determinó mediante el método de concentración por flotación con solución fenol azucarada o de Sheather. Las variables época, sexo y edad se analizaron por la prueba de Chi Cuadrado usando el software estadístico MINITAB.

\section{Resultados y Discusión}

La frecuencia de perros pastores positivos a ooquistes o esporoquistes de Sarcocystis sp. fue de $56.4 \pm 6.7 \%$ (Cuadro 1), encontrándose diferencias significativas en relación a la época del año y al grupo etáreo $(p>0.05)$.

La frecuencia de sarcocistiosis canina hallada en el presente estudio fue elevada, resaltando la gravedad de la enfermedad en las zonas altoandinas del sur del país y reafirmando el rol importante que juega la población canina en la difusión de esta enfermedad. La situación de la sarcocistiosis canina en otros países de la región es mínima. Así se halló un 9.9\% (219/2193) de canes positivos a Sarcocystis en Buenos Aires, Argentina (Fontanarrosa et al., 2006), 2.2\% (6/ 271) en Sao Paulo, Brasil (Oliveira-Sequeira et al., 2002) y $3.6 \%$ (35/972) en Santiago de Chile (López et al., 2006), mientras que en Europa se reportaron tasas más bajas $(0.6 \%$ en República Checa, Dubná et al., 2006 y 2.5\% en España, Martínez-Moreno et al., 2007).

En el Cuadro 1 se muestra la frecuencias de infección encontradas durante el periodo de evaluación, pudiendo observarse una cierta estacionalidad de la enfermedad durante la época lluviosa ( $\mathrm{p}<0.05)$. Las mayores tasas se presentaron entre setiembre y febrero, meses donde hay una mayor pro- 
ducción de forraje y coincide con el empadre y parición de los CSA. Las lluvias van acompañadas de temperaturas promedio de 10 a $15^{\circ} \mathrm{C}$ (SENAMHI, 2005), favorables para la mayor presentación de problemas sanitarios y, sobre todo, de tipo parasitarios. No existen estudios de investigación sobre la estacionalidad de la sarcocistiosis, con excepción del trabajo de Fontanarrosa et al. (2006) quienes encontraron una mayor frecuencia de Sarcocystis al final del verano (febrero-marzo), similar a lo hallado en el presente trabajo.

Estos resultados pueden servir de alerta para considerar y tener en cuenta que la época lluviosa podría ser considerada como de mayor riesgo de infección a Sarcocystis para el ganado altoandino. Además, no se debe de olvidar que los esporozoitos son inmediatamente infectivos, pudiendo permanecer viables por mucho tiempo en condiciones de humedad y baja temperatura (Leguía, 1991). La lluvia y el viento, entre otros aspectos, podrían favorecer la supervivencia y diseminación de los esporoquistes en el medio ambiente.

No se encontró diferencias significativas entre sexos (Cuadro 1). Esto puede ser corroborado por estudios similares, donde el sexo no fue una variable predisponente para Sarcocystis sp. (Fontanarrosa et al., 2006; López et al., 2006).

La frecuencia de Sarcocystis sp. se incrementa a medida que aumenta la edad ( $\mathrm{p}<0.05)$. Fontanarrosa et al. (2006) y López et al. (2006) no encontraron diferencias entre grupos etáreos, aunque siempre se presume que los animales de mayor edad tienen un mayor porcentaje de infección debido al mayor tiempo de exposición. Además, los perros adultos podrían desplazarse más libremente y tener acceso a posibles fuentes de infección (robo de vísceras). Desafortunadamente, aún no se cuenta con un tratamiento ideal para controlar la sarcocistiosis canina, siendo las medidas preventivas la mejor opción para el control de la enfermedad (educación sanitaria, evitar consumo de vísceras y carne infectada, etc.).
Otro aspecto que se debe de investigar es la posibilidad de infección de los perros pastores por el consumo de otras presas infectadas con Sarcocystis sp., como ratones, vizcachas y cuyes silvestres.

\section{Conclusiones}

- La frecuencia de ooquistes y esporoquistes de Sarcocystis sp. en perros pastores de asociaciones alpaqueras del distrito de Maranganí, Cusco fue elevada $(56.4 \pm 6.7 \%)$.

- La mayor frecuencia de Sarcocystis sp. (72.0 $\pm 8.8 \%)$ ocurrió en la época lluviosa $(\mathrm{p}<0.05)$.

- La tasa de infección por Sarcocystis sp. aumentó con la edad $(\mathrm{p}<0.05)$ pero no se afectó con el sexo de los canes.

\section{Agradecimiento}

Un agradecimiento especial a INCAGRO, por el apoyo económico en la realización del presente proyecto, enmarcado dentro del subproyecto "Estrategia de manejo genético, sanitario y nutricional en la expresión de la capacidad genética de llamas para producción de carnes". Así como al Dr. Mario García por el apoyo incondicional en la corrección del presente artículo.

\section{Literatura Citada}

1. Cornejo, R.; A. Chávez; V. Leyva; N. Falcón; S. Panez; D. Ticona. 2007. Relación entre el tamaño de los macroquistes de Sarcocystis aucheniae y su viabilidad en Canis familiares. Rev. Inv. Vet., Perú 18: 76-83.

2. Dubná, S.; I. Langrová; J. Nápravnik; I. Jankovská; J. Vadlejch; S. Pekár; J. Fechtner. 2006. The prevalence of intestinal parasites in dogs from Prague, rural areas, and shelters of the Czech Republic. Vet Parasitol. [Internet] Dispo- 
nible en: www.sciencedirect.com/10.1016/ vetpar2006.11.006

3. Fontanarrosa, M.F.; D. Vezzani; J. Basabe; D.F.Eiras. 2006. An epidemiological study of gastrointestinal parasites of dogs from southern greater Buenos Aires (Argentina): age, gender, breed, mixed infections, and seasonal and spatial patterns. Vet. Parasitol. 136: 283-295.

4. [INIA] Instituto Nacional de Investigación y Extensión Agraria. 2007. Camélidos. [Internet] [acceso 1 junio 2007]. Disponible en: www.inia.gob.pe/ Camelidos/resumen.htm

5. Leguía, G. 1991. Enfermedades parasitarias. En: Avances y perspectivas de los camélidos sudamericanos. FernándezBaca (ed). p 326-362. FAO. Santiago de Chile.

6. Leguía, G.; E. Casas. 1999. Enfermedades parasitarias y atlas parasitológico de camélidos sudamericanos. p 23-30. Editorial De Mar. Lima.

7. López, J.D.; K.V. Abarca; P.M. Paredes; E. Inzunza. 2006. Intestinal parasites in dogs and cats with gastrointestinal symptoms in Santiago, Chile. Rev. Med. Chile 134: 193-200.

8. Martínez-Moreno, F.J.; S. Hernández; E. López-Cobos; C. Becerra; I. Acosta; A. Martínez-Moreno. 2007. Estimation of canine intestinal parasites in Cordova (Spain) and their risk to public health. Vet. Parasitol. 143: 7-13.

9. Mostajo, W. 1983. Sarcocistiosis en alpacas beneficiadas en el camal municipal de Santa Rosa. Tesis de Médico Veterinario Zootecnista. Facultad de Medicina Veterinaria y Zootecnia, Univ. Nacional del Altiplano. Puno. 68 p.

10. Oliveira-Sequeira, T.C.; A.F. Amarante; T.B. Ferrari; L.C. Nunes. 2002. Prevalence of intestinal parasites in dogs from Sao Paulo State, Brazil. Vet. Parasitol. 103: 19-27.

11. [SENAMHI] Servicio Nacional de Metereología e Hidrología. 2005. Oficina de Estadística e informática. Estación Meteorológica de Sicuani, Canchis. 1 p.

12. Soulsby, E.J.L. 1988. Parasitología y enfermedades parasitarias. $7^{\mathrm{ma}}$ ed. $\mathrm{p}$ 681702. Ed. Interamericana. México. 